\title{
MODULE INEQUALITIES FOR QUASIREGULAR MAPPINGS
}

\author{
CABIRIA ANDREIAN CAZACU
}

The problem of finding the $n$-dimensional generalization of the theory of complex analytic functions in the plane gave rise to different important research fields among which the relatively recent theory of quasiregular mappings is one of the most successful. This theory has its roots in the theory of quasiconformal mappings (i.e. quasiconformal homeomorphisms) and in Stoilow's topological theory of analytic functions. It was first developed by Ju. G. Rešetnjak [8] - [10] and by O. Martio, S. Rickman and J. Väisälä [2] - [4].

As in quasiconformality, the module of curve or surface families constitutes a useful instrument in the study of quasiregularity. Several authors, O. Martio, S. Rickman and J. Väisälä [2], E. A. Poleckiü [6], J. Väisälä [11], and others, obtained different generalizations of Grötzsch module inequalities from 2- and $n$-dimensional quasiconformality to quasiregularity. These results permitted one to characterize quasiregular mappings in terms of modules and to establish numerous properties for them.

The purpose of our paper is to prove other module inequalities which improve the above mentioned ones in the following respects:

1. Using the module with weight we need not use global dilatations, and the deduced bounds are sharper. In this way we obtain also equality cases. The results are given for the module with order, too.

2. The inequalities and equalities we prove are valid not only for curves but also for $q$-dimensional surfaces $(q=2, \ldots, n-1)$.

3. They apply to a more general class of mappings than that of the quasiregular mappings, especially because they need no finite global dilatations.

Our method is based on results and techniques we gave in [1], and the equality cases we obtain extend the module transformation formula of [1] for quasiregular or more general mappings.

Our considerations may be naturally applied to capacity, which is closely related to module [11]. 


\section{Preliminaries}

We use the same definitions and notations as in [1] and [2].

We work in $R^{n}$ with the Lebesgue measure and integral and use the following abbreviations: a.e. for almost everywhere; a. for almost; 1. for local or locally; $q$ - for $q$-dimensional, $q=1,2, \ldots, n$, in expressions like $q$-measure, $q$-element, $q$-surface (the case $q=1$ in the last expression corresponding to the curves). If $E$ is a $q$-measurable set in $R^{q}, q=$ $1, \ldots, n$, its $q$-measure will be designated by $\mu_{q}(E)$.

$G$ will be a domain in $R^{n}$ and $f: G \rightarrow R^{n}$ a non-constant, continuous mapping. The images under $f$ or different notions related to the images will be designated by a $*$. We shall write $x^{*}=f(x)$ for $x \in G ; G^{*}=f(G)$; $C^{*}=f(C)$ for $C$ a $q$-surface in $G$; and $d \sigma_{n}, d \sigma_{n}^{*}$ and $d \sigma_{q}, d \sigma_{q}^{*}$ for the $n$-dimensional volume element in $G$ and $G^{*}$ or the $q$-dimensional area element on $C$ and $C^{*}$, respectively.

1.1. Local dilatations in regular points. We begin with the following

$\mathrm{D}$ e finition 1. A point $x \in G$ is regular with respect to $f$, if $f$ is differentiable at $x$ and has non-vanishing Jacobian $J(x, f)=J_{n}=$ $d \sigma_{n}^{*} / d \sigma_{n}$.

We suppose $f$ to be sense-preserving, hence $J(x, f) \geq 0$.

Let $f^{\prime}(x): R^{n} \rightarrow R^{n}$ be the differential of $f$ at $x$ and $A(f, x)$ the affine transformation associated to $f$ at $x$,

$$
A(f, x): X \in R^{n} \mapsto f(x)+f^{\prime}(x)(X-x) \in R^{n} .
$$

It is well known that $A(f, x)$ transforms a ball $B^{n}(x, 1)$ with the center $x$ and the radius 1 in the "characteristic ellipsoid" $E^{n}\left(x^{*}, f^{\prime}(x)\right)$ with the center $x^{*}$ and the semi-axes $a_{1} \geq \ldots \geq a_{n}$, where

$$
\begin{gathered}
a_{1}=\left\|f^{\prime}(x)\right\|=\max _{|h|=1}\left|f^{\prime}(x) h\right|, \quad a_{n}=l\left(f^{\prime}(x)\right)=\min _{|h|=1}\left|f^{\prime}(x) h\right| \\
\left(h=\left(h_{1}, \ldots, h_{n}\right) \in R^{n}, \quad|h|=\left(\sum_{j=1}^{n} h_{j}^{2}\right)^{1 / 2}\right) \quad \text { and } \\
J(x, f)=\operatorname{det} f^{\prime}(x)=a_{1} \ldots a_{n} .
\end{gathered}
$$

One defines the inner dilatation

$$
H_{I}\left(f^{\prime}(x)\right)=\frac{J(x, f)}{l\left(f^{\prime}(x)\right)^{n}}=\frac{a_{1} \ldots a_{n-1}}{a_{n}^{n-1}}=\frac{\mu_{n}\left[E^{n}\left(x^{*}, f^{\prime}(x)\right)\right]}{\mu_{n}\left[B^{n}\left(x^{*}, l\right)\right]},
$$

the outer dilation

$$
H_{o}\left(f^{\prime}(x)\right)=\frac{\left\|f^{\prime}(x)\right\|^{n}}{J(x, f)}=\frac{a_{1}^{n-1}}{a_{2} \ldots a_{n}}=\frac{\mu_{n}\left[B^{n}\left(x^{*},\left\|f^{\prime}(x)\right\|\right)\right]}{\mu_{n}\left[E^{n}\left(x, f^{\prime}(x)\right)\right]}
$$

and the linear dilatation $H=a_{1} / a_{n}$ of $f$ at $x$ (or of $f^{\prime}(x)$ ). 
Besides these dilatations we considered q-dimensional dilatations $([1, \S 2])$ in the transformation formula for the module of $q$-surface families. Such a dilatation was introduced by $R$. Nevanlinna in [5] for $m=n$ and a family of concentric $(n-1)$-spheres.

Let $C$ be a $q$-surface through $x$ with a tangent $q$-plane $\Pi$ at $x$. The restriction $f \mid \Pi$ is $q$-differentiable at $x$ (in an evident sense) and has $A(f, x) \mid \Pi$ as the associated affine mapping with the Jacobian $J_{q}=$ $d \sigma_{q}^{*} / d \sigma_{q}$. We define the $q$-dimensional dilatation of $f$ at $x$ with respect to $C$ or $\Pi$ and with respect to $m>0$ (a number equal to the Fuglede order of the module in the transformation formula and equal to $n$ in the usual case)

$$
d=d_{f, C, m}(x)=\frac{J_{q}^{\frac{m}{q}}}{J_{n}} .
$$

We drop the indices $f, C$ or $m$ which are evident, in particular $m$ if $m=n$.

The geometric interpretation of $d$ is elementary: $A(f, x)$ transforms the $q$-ball $B_{\Pi}^{q}(x, 1)=B^{n}(x, 1) \cap \Pi$ in a $q$-ellipsoid $E_{\Pi}^{q}\left(x^{*}, f^{\prime}(x)\right)$ with the semi-axes $v_{1} \geq \ldots \geq v_{q},[1, p$. 97]. Then

$$
d=\frac{\left(v_{1} \ldots v_{q}\right)^{\frac{m}{q}}}{a_{1} \ldots a_{n}}
$$

and if one puts

$$
H_{I, q}^{-(m-q)}\left(f^{\prime}(x)\right)=\frac{\left(a_{n-q+1} \ldots a_{n}\right)^{\frac{m-q}{q}}}{a_{1} \ldots a_{n-q}}, \quad H_{O, q}^{m-q}\left(f^{\prime}(x)\right)=\frac{\left(a_{1} \ldots a_{q}\right)^{\frac{m-q}{q}}}{a_{q+1} \ldots a_{n}},
$$

it follows

$$
H_{I, q}^{-(m-q)} \leq d \leq H_{O, q}^{m-q} .
$$

More precisely, $H_{I, q}$ and $H_{O, q}$ should be written with an index $m$, too. If $q=1$ and $m=n$, then $H_{I, 1}^{n-1}=H_{I}, H_{O, 1}^{n-1}=H_{O}$.

Usually in this paper $f$ will be regular $n$-a.e. in $G$ and through $n$-a. every point $x \in G$ will pass one and only one $q$-surface $C$ of the considered family $C=\{C\}$ such that we shall have a function $d$ defined $n$-a.e. in $G$, which expresses the dilatation of $f$ with respect to $C$ better than the global constants generally used.

1.2. Quasiregular mappings. We recall the following characterization $([2]-[4])$.

Definition 2. A mapping $f: G \rightarrow R^{n}$ is quasiregular if 
1) $f$ is $\mathrm{A} \mathrm{CL}^{n}$ (absolutely continuous on lines with 1. $L^{n}$-integrable partial derivatives) in $G$, and

2) there exists a constant $K, 1 \leq K<\infty$, such that

$$
\left\|f^{\prime}(x)\right\|^{n} \leq K J(x, f)
$$

holds for a. every $x \in G$.

According to Ju. G. Rešetnjak [9], [10] a quasiregular mapping is either constant or sense-preserving, discrete and open; it is differentiable $n$-a.e. in $G$ and has the property $(\mathrm{N})$.

The branch set $B_{f}$ of $f$ has a dimension $\leq n-2$.

One defines the global dilatations

$$
K_{o}(f)=\underset{x \in G}{\operatorname{ess} \sup } H_{O}\left(f^{\prime}(x)\right) \text { and } K_{I}(f)=\operatorname{ess} \sup _{x \in G} H_{I}\left(f^{\prime}(x)\right)
$$

for which

$$
\left\|f^{\prime}(x)\right\|^{n} \leq K_{o}(f) J(x, f), \quad J(x, f) \leq K_{I}(f) l\left(f^{\prime}(x)\right)^{n}
$$

$n$-a.e. in $G$ and the maximal dilatation $K(f)=\max \left[K_{I}(f), K_{O}(f)\right]$.

Similarly one defines $K_{O, q}(f), K_{I, q}(f)$ starting from $H_{O, q}$ and $H_{I, q}$, respectively.

Definition 3. A quasiregular mapping is $K$-quasiregular for each constant $K \geq K(f)$.

Definition 4. A homeomorphism $f: G \rightarrow G^{*}$ is called a $(K-)$ quasiconformal mapping if it is $(K$-)quasiregular.

If $m=n$ and $f$ is $K$-quasiregular, we have $K_{O, q}(f), K_{I, q}(f) \leq$ $\min \left(K^{2 / n}, K^{1 /(n-q)}\right)$.

1.3. Module inequalities for quasiregular mappings. We shall work with the following definition of the module (Ahlfors and Beurling, Fuglede, Ohtsuka):

Definition 5. Let $q$ be an integer, $1 \leq q<n, C=\{C\}$ a family of $q$-surfaces in the domain $G, m$ a positive number, and $\pi$ a function which is defined $n$-a.e. in $G$, finite, positive and measurable. We denote by $\mathfrak{A}(C)$ the family of all functions $\varrho$ with the following properties: $\varrho$ is defined in $G$, non-negative and measurable; for every surface $C$ on which the measure and the integral are defined, we have

Further we write

$$
\int_{C} \varrho^{q} d \sigma_{q} \geq 1 \text {. }
$$

$$
A_{Q ; \pi, m}(G)=\int_{G} \pi \varrho^{m} d \sigma_{n}
$$


The module of order $m$ and with weight $\pi$ of the family $C$ is by definition

$$
M_{\pi, m}(G)=\inf _{\varrho \in \mathfrak{U}(C)} A_{\varrho ; \pi, m}(G)
$$

We drop the index $m$ if $m=n$, and $\pi$ if $\pi=1$.

One knows that the $K$-quasiconformal mappings satisfy the Grötzsch inequalities

$$
K^{-1} M(C) \leq M\left(C^{*}\right) \leq K M(C)
$$

for each curve family $G$ in $G$ and can be characterized by this property (or by weaker properties, if one uses only certain curve families and only a part of the double inequality above). More precisely,

$$
K_{o}(f)=\sup _{c} \frac{M(C)}{M\left(C^{*}\right)}, \quad K_{I}(f)=\sup _{c} \frac{M\left(C^{*}\right)}{M(C)}
$$

for every curve family $C$ in $G$. Thus,

$$
K_{o}(f)^{-1} M(G) \leq M\left(C^{*}\right) \leq K_{I}(f) M(C) .
$$

The problem of extending such inequalities to non-constant quasiregular mappings has been studied by several authors. If we renounce the injectivity of the mapping it becomes natural to introduce in these inequalities a factor which expresses the multiplicity.

One uses for instance the multiplicity functions

$$
N\left(x^{*}, f, A\right)=\operatorname{card} f^{-1}\left(x^{*}\right) \cap A,
$$

where $A \subset G$ and $x^{*} \in R^{n}$ and

$$
N(f, A)=\sup _{x^{*} \in R^{n}} N\left(x^{*}, f, A\right) .
$$

Let us recall some of the results obtained for a non-constant quasiregular mapping $f$ :

Theorem of Martio-Rickman-Väisälä ([2, 3.2]).

$$
M(C) \leq N(f, A) K_{o}(f) M\left(C^{*}\right)
$$

for each Borel set $A \subset G$ with $N(f, A)<\infty$ and each family of paths $C$ in $A$.

Poleckil's Theorem ([6, Theorem 2]).

$$
M\left(C^{*}\right) \leq \frac{K}{N(f, A)} M(C)
$$

for $f K$-quasiregular, $A$ a normal domain of $f$ in $G$ (i.e. a bounded domain $A$ such that $\mathrm{cl} A \subset G$ and $f(\partial A)=\partial f(A)), C^{*}$ a family of injective paths 
in $f(A)$ and $C$ the set of all paths in $A$ with $f \circ C \subset C^{*}$. (As in [11], $f \circ C \subset C^{*}$ means that $f \circ C=C^{*} \mid I, I$ a subinterval of the definition interval of $\left.C^{*}\right)$. According to Väisälä one can put $K_{I}(f)$ instead of $K$ in (5).

Väis älä's Theorems ([11, §3]).

$$
M\left(\Gamma^{\prime}\right) \leq \frac{K_{I}(f)}{\mathfrak{m}} M(\Gamma)
$$

where either

I. $\Gamma$ is a path family in $G, \Gamma^{\prime}$ a path family in $R^{n}$ and $\mathrm{m}$ a positive integer with the following properties: There is a set $E_{0} \subset G$ of measure zero such that for every path $\beta: I \rightarrow R^{n}$ in $\Gamma^{\prime}$ there are paths $\alpha_{1}, \ldots, \alpha_{\mathfrak{m}}$ in $\Gamma$ such that $f \circ \alpha_{i} \subset \beta, i=1, \ldots, \mathfrak{m}$, and such that for each $x \in G \backslash E_{0}$ and $t \in I, \operatorname{card}\left\{i \mid \alpha_{i}(t)=x\right\} \leq 1$;

or

II. $\Gamma$ is a path family in $G, \Gamma^{\prime}=\Gamma^{*}=f(\Gamma)$, $\mathrm{m}$ is a positive integer and $f$ winds every path in $\Gamma \mathrm{m}$ times around itself $([11, \mathrm{p} .9])$.

\section{Some new module inequalities}

2.1. In this paragraph we shall try to improve the results mentioned above by means of the module with weight. This device permitted us to obtain in [1], under certain conditions concerning the considered family $C$ of $q$-surfaces and the applied homeomorphism $f: G \rightarrow G^{*}$, the module transformation formula

$$
M_{\pi^{*}, m}\left(C^{*}\right)=M_{\pi d^{-1}, m}(C), \quad \pi^{*}=\pi \circ f^{-1},
$$

i.e., an equality instead of Grötzsch inequalities.

Let us recall these conditions given in [1]:

a) $f$ is differentiable $n$-a.e. in $G$.

$\beta$ ) $f$ and $f^{-1}$ satisfy the condition (N) (or equivalently $f$ is 1 . bimeasurable in $G$ ).

$\gamma .1)$ The family $C$ covers $G$ with the possible exception of a set of measure zero such that the function $d=d_{f, c, m}$ is well defined $n$-a.e. in $G$, positive, finite and measurable.

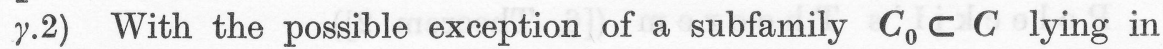
a subset of $G$ with $n$-measure zero, measure and integral are well defined on $C$ and $C^{*}=f(C) ; f \mid C$ and $f^{-1} \mid C^{*}$ satisfy the condition $(\mathrm{N}) ; f$ is differentiable $q$-a.e. on $C ; J_{q}$ exists $q$-a.e. on $C$ and is l. integrable on $C$; and for each function $h^{*}$, which is defined $q$-a.e. on $C^{*}$, non-negative, finite and measurable, the transformation formula 


$$
\int_{C^{*}} h^{*} d \sigma_{q}^{*}=\int_{C}\left(h^{*} \circ f\right) J_{q} d \sigma_{q}
$$

holds. Evidently, if the constants $K_{O, q}$ and $K_{I, q}$ are finite, in particular if $f$ is quasiconformal, (7) implies

$$
K_{O, q}^{-(m-q)}(f) M_{\pi, m}(C) \leq M_{\pi^{*}, m}(C) \leq K_{I, q}^{(m-q)}(f) M_{\pi, m}(C) .
$$

(More general conditions about the family $C$ and the exceptional family $C_{0}$ are given in [1].)

In order to obtain module inequalities for non-constant quasiregular mappings we shall consider not only the dilatations $d$ corresponding to the family $C$ but local multiplicity functions, too:

$$
\begin{aligned}
& N_{n}^{*}\left(x^{*}\right)=N\left(x^{*}, f, G\right), \quad N_{n}=N_{n}(x)=N_{n}^{*}(f(x)), \\
& N_{q}=N_{q}(x)=N(f(x), f, C) \text { for } x \in C,
\end{aligned}
$$

and

$$
v=v_{f, C, m}(x)=\frac{N_{q}^{\frac{m}{q}}}{N_{n}} .
$$

We shall use the transformation formula for integrals ([7, p. 364, Theorem 3])

$$
\int_{G^{*}} H^{*}\left(x^{*}\right) N\left(x^{*}, f, G\right) d \sigma_{n}^{*}=\int_{G}\left(H^{*} \circ f\right)(x) J_{n}(x) d \sigma_{n},
$$

true if $f$ is a continuous, bounded, $n$-a.e. differentiable mapping, which satisfies the condition $(\mathrm{N})$ and has a l. integrable Jacobian, and if $H^{*}$ is a non-negative, finite and measurable function $n$-a.e. in $G^{*}$.

Theorem 1. Let $G$ be a q-surface family in $G, m>0$, and $f: G \rightarrow G^{*}$ a mapping such that

$\left.\alpha_{1}\right) f$ is differentiable n-a.e. in $G$ and $J_{n}$ is l. integrable in $G$.

$\left.\beta_{1}\right) f$ satisfies the condition $(\mathrm{N})$ in $G$ and $N_{n}(x)<\infty$ n-a.e. in $G$. $\left.\gamma_{1} .1\right)$ as $\gamma .1$ ) above.

$\left.\gamma_{1} .2\right)$ With the possible exception of a subfamily $C_{0} \subset C$ lying in a subset of $G$ with n-measure zero, measure and integral are well defined on $C$ and $C^{*}=f(C)$; $f$ is q-a.e. differentiable on $C, J_{q}$ is $l$. integrable on $C, f \mid C$ satisfies $(\mathrm{N})$ and $N_{q}(x)<\infty$ q-a.e. on $C$; and for $C$ and each function $h^{*}$ as in $\gamma .2$ ) above

$$
\int_{C^{*}} h^{*}\left(x^{*}\right) N_{q}\left(x^{*}, f, C\right) d \sigma_{q}^{*}=\int_{C}\left(h^{*} \circ f\right)(x) J_{q}(x) d \sigma_{q} .
$$


$\left.\delta_{1}\right)$ The functions $\pi^{*}, \pi=\pi^{*} \circ f$ and $v$ are well-defined $n$-a.e. in $G^{*}$ or $G$, respectively, they are positive, finite and measurable.

Then

$$
M_{\pi d^{-1}, m}(C) \leq M_{\pi^{*}, m}\left(C^{*}\right) .
$$

Proof. Let $\varrho^{*}$ be a function in $\mathfrak{A}\left(C^{*}\right)$ and $C \in C \backslash C_{0}$. Since

$$
1 \leq \int_{C^{*}} \varrho^{* q} d \sigma_{q}^{*}=\int_{C}\left(\varrho^{* \circ f}\right)^{q} \frac{J_{q}}{N_{q}} d \sigma_{q}
$$

the function

$$
\varrho= \begin{cases}\left(\varrho^{* \circ f)}\left(\frac{J_{q}}{N_{q}}\right)^{\frac{1}{q}},\right. & \text { if } 0<J_{q}, N_{q}<\infty \\ +\infty & \text { otherwise }\end{cases}
$$

belongs to $\mathfrak{A}(\boldsymbol{C})$.

On the other hand

$$
A_{\varrho^{*} ; \pi^{*}, m}\left(G^{*}\right)=\int_{G^{*}} \pi^{*} \varrho^{* m} d \sigma_{n}^{*}=\int_{G} \pi \varrho^{m} v d^{-1} d \sigma_{n}=A_{\varrho ; \pi d^{-1} v, m}(G) .
$$

As $\varrho^{*}$ is an arbitrary function in $\mathfrak{A}\left(C^{*}\right)$ one deduces immediately (12).

Consequence 1. Suppose that (for $m$, and $C$ ) we have $K_{o, q}(f)<\infty$ and $N(f, G)=\sup _{x \in G} N_{n}(x)<\infty$. Therefore the inequalities $d^{-1} \geq K_{O, q}^{-(m-q)}$ and $v \geq 1 / N(f, G)$ hold $n$-a.e. in $G$. Hence (12) implies

$$
M_{\pi d^{-1}, m}(C) \leq N(f, G) M_{\pi *, m}\left(C^{*}\right)
$$

and

$$
M_{\pi, m}(G) \leq N(f, G) K_{O, q}^{(m-q)}(f) M_{\pi^{*}, m}\left(C^{*}\right) .
$$

In particular, for

$$
q=1, m=n, \pi=\pi^{*}=1, \text { one obtains (4). }
$$

(We can deduce similar inequalities by using $v \geq \operatorname{ess~}_{\inf }{ }_{x \in G} N_{q}(x)^{m / q} / N(f, G)$ instead of $v \geq 1 / N(f, G)$.)

Remark 1. The inequality (12) holds even if one renounces the hypothesis that one and only one $C$ passes through $n$-a. every $x \in G$, and if (9) is replaced by

$$
v(x)=\frac{\inf _{C} N_{q}(x)^{\frac{m}{q}}}{N_{n}(x)}
$$


where inf is taken over all $C$ which pass through $x$. Consequence 1 can then be reformulated.

2.2. Generally there is no equality in (12). However, on certain conditions concerning $f$ and the family $C$ one has the transformation formula

$$
M_{\pi d^{-1},, m}(C)=M_{\pi^{*}, m}\left(C^{*}\right) \text {. }
$$

We shall give some results in this sense:

$\mathrm{Th}$ e or e m 2. Assume, in addition to the hypotheses of Theorem 1, that there exists an extremal metric $\tilde{\varrho} \in \mathfrak{A}(C)$ for $M_{\pi d^{-1} v, m}(C)$, such that there is a function $\varrho^{*}: G^{*} \rightarrow R$ for which

$$
\varrho^{* \circ f}=\tilde{\varrho}\left(\frac{N_{q}}{J_{q}}\right)^{\bar{q}}
$$

n-a.e. in G. Then (13) holds.

Proof. The function $\varrho^{*}$ belongs to $\mathfrak{A}\left(C^{*}\right)$. Indeed, for each $C^{*}$ such that there exists a non-exceptional $q$-surface $C$ with $f(C)=C^{*}$, we have

Therefore

$$
1 \leq \int_{C} \tilde{\varrho}^{q} d \sigma_{q}=\int_{C^{*}} \varrho^{* q} d \sigma_{q}^{*} .
$$

$$
M_{\pi^{*}, m}\left(C^{*}\right) \leq A_{\varrho^{*} ; \pi^{*}, m}\left(G^{*}\right)=A_{\tilde{\varrho} ; \pi d^{-1} v, m}(G)=M_{\pi d^{-1}, m}(C) .
$$

2.3. We shall now deduce a module transformation formula (13) (by means of the module representation formula [1]) for a family of $q$-surfaces $C=\left\{C_{\eta}\right\}_{\eta \in \Re}$ depending on a parameter $\eta$ such that $G$ covers $G$ when $\eta$ describes an $(n-q)$-dimensional domain $\Re$ in $R^{n-q}$. Suppose that $m>q$ and $\pi$ is as in Definition 5. Under the regularity conditions given in $[1$, p. 89 , Theorem 1$]$, we proved the module representation formula

$$
M_{\pi, m}(C)=\int_{\Re} L_{\pi^{-1}, m}^{-1}\left(C_{\eta}\right) d \eta .
$$

Here $d \eta$ represents the $(n-q)$-element in $\Re$ and

$$
L_{\pi^{-1}, m}\left(C_{\eta}\right)=\left[\int_{C_{\eta}}\left(\pi^{-1} j^{-1}\right)^{\frac{q}{m-q}} d \sigma_{q}\right]^{\frac{m-q}{q}},
$$

$j$ being a positive, finite and measurable function $n$-a.e. in $G$, such that $d \sigma_{n}=j d \sigma_{q} d \eta$ 
Th e or e m 3. Let $f: G \rightarrow G^{*} \subset R^{n}$ be a mapping such that $G^{*}$ is a domain and let $C=\left\{C_{\eta}\right\}_{\eta \in \Re}$ and its image $C^{*}=\left\{C_{\eta^{*}}^{*}\right\}_{\eta^{*} \in} \Re^{*}$ be families as above; here $\Re$ and $\Re^{*}$ are domains in $R^{n-q}$. Assume that $C_{\eta^{*}}^{*}=f\left(C_{\eta}\right)$ where $\eta^{*}=T(\eta), T$ being a continuous sense-preserving surjection $\Re \rightarrow \Re^{*}$. Further assume that $f$ and $T$ fulfil the regularity conditions $\left.\left.\alpha_{1}\right), \beta_{1}\right) \gamma_{1}$ ) necessary in the subsequent calculations (see transformation formula [1, p. 92]), that $N_{n-q}(\eta)=N\left(\eta^{*}, T, \Re\right)<\infty$ for $(n-q)$-a. every $\eta$ in $\Re, N_{q}(x)=$ $N\left(x^{*}, f, C_{\eta}\right)<\infty$ for $q-a$. every $x$ in $C_{\eta}$ and $(n-q)$-a. every $\eta$ in $\Re$, and $N_{n}(x)=N_{q}(x) N_{n-q}(\eta), x \in C_{\eta}$, for $n$-a. every $x$ in $G$, all these functions $N$ as well as $v=N_{q}^{m / q} / N_{n}=N_{q}^{(m-q) / q} / N_{n-q}$ being measurable, that $m>q$, and that $\pi^{*}$ and $\pi=\pi^{*} \circ f$ verify the usual properties of the weight. Then one has again

$$
M_{\pi *, m}\left(C^{*}\right)=M_{\pi d^{-1} v, m}(C) .
$$

Proof. It suffices to make a direct calculation based on (14). One has

with

$$
M_{\pi^{*}, m}\left(C^{*}\right)=\int_{\Re^{*}} L_{\pi^{*-1}, m}^{-1}\left(C_{\eta^{*}}^{*}\right) d \eta^{*}
$$

$$
L_{\pi^{*-1}, m}\left(C_{\eta^{*}}^{*}\right)=\left[\int_{C_{\eta^{*}}^{*}}\left(\pi^{*-1} j^{*-1}\right)^{\frac{q}{m-q}} d \sigma_{q}^{*}\right]^{\frac{m-q}{q}} .
$$

Since $j^{*-1} \circ f=j^{-1}\left(J_{q} / J_{n}\right) \mathfrak{\Im}_{n-q}$, where $\mathfrak{\Im}_{n-q}(\eta)=d \eta^{*} / d \eta$, one obtains

Therefore

$$
L_{\pi^{*-1}, m}\left(C_{\eta^{*}}^{*}\right)=\Im_{n-q}(\eta) L_{\pi^{-1} d N_{q}^{-(m-q) / q}, m}\left(C_{\eta}\right)
$$

$$
M_{\pi^{*}, m}\left(C^{*}\right)=\int_{\Re} L_{\pi^{-1} d N_{q}^{-(m-q) / q}, m}^{-1}\left(C_{\eta}\right) N_{n-q}^{-1}(\eta) d \eta=M_{\pi d^{-1}, m}(G)
$$

If, under the conditions of Theorem $3, N_{q}$ and $N_{n-q}$ are constants, then

$$
M_{\pi^{*}, m}\left(C^{*}\right)=v M_{\pi d^{-1}, m}(C) \text {. }
$$

Examples. 1.1. We set

$$
G=\{z=x+i y|1<| z \mid<\varrho\} ; \Re=\{\eta \in R \mid 0 \leq \eta \leq 2 \pi\} ;
$$

$C_{\eta}$ is the radial segment in $G: z=|z| e^{i \eta} ; f$ is the function $z \mapsto z^{N}$. Then $q=1, n=2, d=1, v=1 / N$, and we have $M\left(C^{*}\right)=$ $M(C) / N$. (Theorem I of Väisälä gives here $M\left(C^{*}\right) \leq M(C) / N^{*}$ ) 
1.2. $G$ and $f$ as above; $\Re=\{\eta \in R \mid 1<\eta<\varrho\} ; \quad C_{\eta}=$ $\{z|| z \mid=\eta\}$. Then $v=N$ and $M\left(C^{*}\right)=N M(C)$. (Theorem II of Väisälä gives now $M\left(C^{*}\right) \leq N M(C)$.)

2. $G$ is a torus in $R^{3}$

$X=\left(r_{0}+r \cos \eta\right) \cos \xi, Y=\left(r_{0}+r \cos \eta\right) \sin \xi$ and $Z=r \sin \eta$, $0<r<r_{0}, \quad \eta \in \Re=[0,2 \pi], \quad \xi \in[0,2 \pi] ; \quad C$ is the family of parallel circles $C_{\eta}$ corresponding to $\eta=$ const. and $f$ carries each $C_{\eta}$ in $C_{\eta^{*}}, \eta^{*}=T(\eta)$, winding each $C_{\eta} \mathfrak{m}$ times around itself and each meridian circle $N$ times around itself. Formula (13) applies again, though $n=2$, for $q=1, N_{q}=\mathfrak{m}, N_{n-q}=N$; hence $v=\mathfrak{m}^{m-1} / N$.

2.4. Remark 2. The results given in Theorems $1-3$ have a natural application to non-constant quasiregular mappings. Indeed many of the assumptions of these theorems are in this case satisfied, with the result that these theorems simplify in an obvious manner.

2.5. The method applied in this paragraph permits one to precise also the inequalities (5) and (6). For instance, one obtains by a slight adaptation of the original authors' proofs [11], [6]:

The or e m 4. Under the hypotheses of Väisälä's theorems $I$ and II in 1.3 (in particular of Poleckiü's theorem in 1.3) we have

$$
M\left(\Gamma^{\prime}\right) \leq \frac{1}{\mathfrak{m}} M_{d^{-1}}(\Gamma)
$$

(or

$$
M\left(C^{*}\right) \leq \frac{1}{N(f, A)} M_{d^{-1}}(C)
$$

respectively).

2.6. Remark 3. One establishes equality cases combining Theorems 1 and 4. For instance, if the hypothesis of Poleckii's Theorem and of Theorem 1 are simultaneously fulfilled, then $M\left(C^{*}\right)=M_{d^{-1}}(C)$.

\section{References}

[1] Andreitan Cazacu, C.: Some formulae on the extremal length in $n$-dimensional case. - Proceedings of the Romanian-Finnish Seminar on Teichmüller spaces and quasiconformal mappings [Brasov, 1969], Publishing House of the Academy of the Socialist Republic of Romania, 1971, 87-102. 
[2] MARTIO, O., S. RICKMAN, and J. VäISÄLÄ: Definitions for quasiregular mappings. - Ann. Acad. Sci. Fenn. Ser. A I 448, 1969, 1-40.

[3] - - Distortion and singularities of quasiregular mappingsీ. - Ibid. 465, $1970,1-13$.

[4] - - Topological and metric properties of quasiregular mappings. - Ibid. $488,1971,1-31$.

[5] Nevanlinna, R.: A remark on differentiable mappings. - Michigan Math. J. (3) $1,1955,53-57$.

[6] Роцескlĭ,Е. А. [Е. А. Полецкий]: Метод модулей для негомеоморфных квазиконформных отображений - Mat. Sb. 83, 1970, 261-272.

[7] RADó, T., and P. V. REICHELDERFER: Continuous transformations in analysis. Springer-Verlag, Berlin-Göttingen-Heidelberg, 1955.

[8] RešEtNJAK, JU. G. [Ю. Г. Решетняк]: Оценки модуля непрерывности для некоторых отображений. - Sibirsk. Mat. Ž. 7, 1966, 1106-1114.

[9] -»- Пространственные отображения с ограниченным искажением. - Ibid. 8, $1967,629-658$.

[10] -»- Об условии ограничености индекса для отображений с огранитенным искажением. - Ibid. 9, 1968, 368-374.

[11] VÄISÄL $\ddot{A}$, J.: Modulus and capacity inequalities for quasiregular mappings. . Ann. Acad. Sci. Fenn. Ser. A I 509, 1972, 1-14.

University of Bucharest

Faculty of Mathematics and Mechanics

Bucharest

Romania

Received 5 September 1975 